\title{
Real-time magnetic resonance imaging of fluidized beds with internals
}

\section{Journal Article}

\section{Author(s):}

Penn, Alexander (1); Boyce, Christopher M.; Conzelmann, Nicholas (1); Bezinge, Gaëtan; Prüssmann, Klaas P.; Müller, Christoph R.

Publication date:

2019-04-28

\section{Permanent link:}

https://doi.org/10.3929/ethz-b-000325567

\section{Rights / license:}

Creative Commons Attribution-NonCommercial-NoDerivatives 4.0 International

\section{Originally published in:}

Chemical Engineering Science 198, https://doi.org/10.1016/j.ces.2018.12.041

\section{Funding acknowledgement:}

153290 - Advancement of magnetic resonance imaging and discrete element models to probe the dynamics of fluidised beds with unprecedented spatial and temporal resolution (SNF) 


\section{Real-time magnetic resonance imaging of fluidized beds with internals ${ }^{\dagger}$}

Alexander Penn ${ }^{1.2, *}$, Christopher M. Boyce ${ }^{2.3}$, Nicholas Conzelmann ${ }^{2}$, Gaëtan Bezinge ${ }^{2}$, Klaas P. Pruessmann $^{1}$ and Christoph R. Müller ${ }^{2, *}$

${ }^{1}$ Institute for Biomedical Engineering, ETH Zurich and University of Zurich

${ }^{2}$ Department of Mechanical and Process Engineering, ETH Zurich

${ }^{3}$ Department of Chemical Engineering, Columbia University

*Corresponding authors: apenn@,ethz.ch and muelchri@ethz.ch

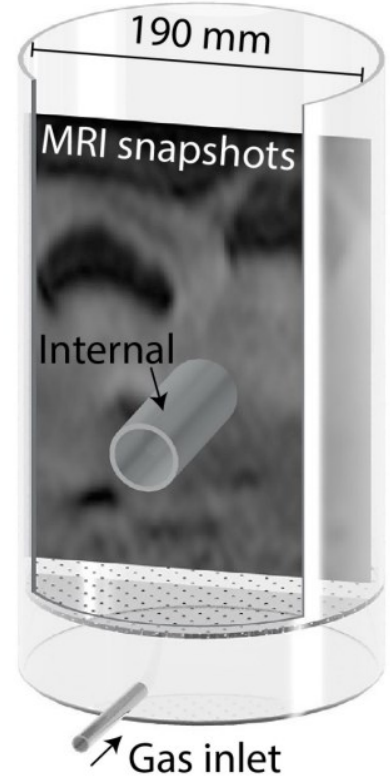

Gas Bubble Positions

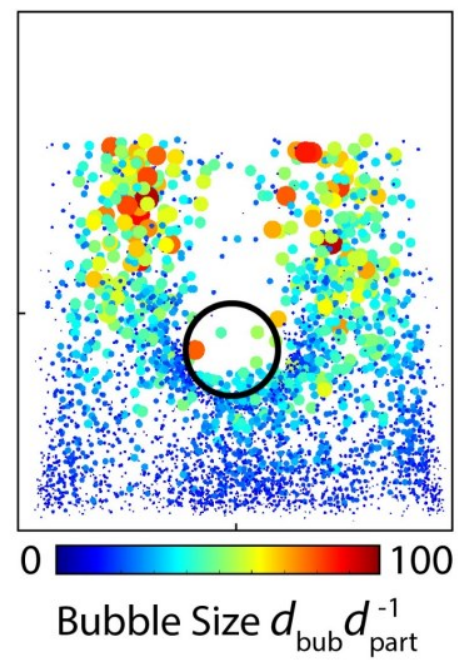

Particle Velocity Fluctuations

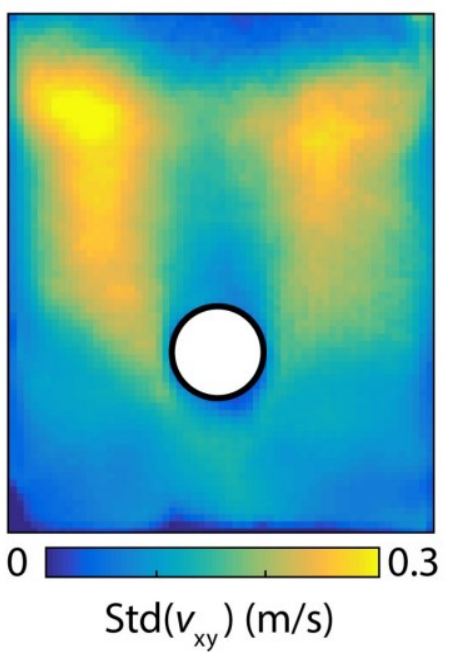

\section{Abstract}

The effect of internals on the fluidization dynamics in three-dimensional (3D) cylindrical fluidized beds was studied using real-time magnetic resonance imaging. Instantaneous snapshots of particle velocity and particle position were acquired for gas velocities $U$ below and above the minimum fluidization velocity $U_{\mathrm{mf}}$. Below $U_{\mathrm{mf}}$, we found local fluidization and gas bubbling in areas adjacent

\footnotetext{
${ }^{\dagger}$ This is an accepted preprint of the article published by the authors. The published version can be found on the webpage of Chemical Engineering Science at https://doi.org/10.1016/j.ces.2018.12.041
} 
to an inserted horizontal tube, likely caused by the gas locally exceeding $U_{\mathrm{mf}}$ as it flows around the insert. Above $U_{\mathrm{mf}}$, the presence of the insert in the bed affected the fluidization dynamics. The wake region above the insert exhibited a lower average particle velocity and reduced velocity fluctuations as well as a substantially reduced number and size of gas bubbles as compared to a reference bed without internals. The extent of this wake region was found to be approximately as wide as the insert, and it extended from the insert to the free surface of the bed. Moreover, the presence of internals also affected the regions below the insert by showing an increased rate of bubble formation directly below the inserted tube. The present work can provide relevant information on the design of fluidized beds with internals. Moreover, the data gathered in this work can be used to validate the accuracy of 3D numerical models of gas-solid systems.

\section{Introduction}

Fluidized bed reactors are used in a variety of industries such as energy conversion, pharmaceuticals and food processing (Senadeera et al., 2003). Fluidized beds allow a comparatively uniform temperature distribution (when compared to packed beds) because particles are dynamic, avoiding the formation of hot spots. The main drivers for particle motion are gas bubbles, yet if they grow too large, the mass and heat transfer characteristics decrease. In this context, internals can be used as they have shown to prevent bubbles from growing excessively large (Baskakov et al., 1973). However, it has also been reported that internals affect the fluidization dynamics within the reactor. The reported effects include (a) the formation of defluidized zones in the wake of a tube (also referred to as de-fluidized caps) (Hager and Schrag, 1976; Rao and Muller, 2013), and (b) the preferential formation of gas bubbles in the vicinity of the obstacles, affecting the bubble distribution in the bed (Glass and Harrison, 1964). While locally de-fluidized zones can lead to hot spots and depleted solid phase remaining in the reactor (Noack, 1970), an increase of the bubble sizes can lower the conversion efficiency due to reduced mixing between the gas in the bubble and particulate phase (Kunii and Levenspiel, 1991). It is hence of fundamental importance to understand how internals in fluidized beds affect fluidization hydrodynamics.

Traditionally, the dynamics of three-dimensional (3D) fluidized beds have been studied experimentally by placing probes into the bulk (Werther and Molerus, 1973) or onto the surface of heat exchanger tubes (Yasuo et al., 1988). Furthermore, optical measurements in spatially reduced, i.e. pseudo-2D systems, have been used (Laverman et al., 2008; Müller et al., 2007). In addition, it 
is possible to track the motion of an individual radioactive tracer particle using positron emission particle tracking (PEPT) (Van de Velden et al., 2008). Extensive temporal averaging will lead to a flow map. While X-ray imaging allows to record 2D projections of the time averaged solid fraction inside a 3D system (Franka and Heindel, 2009), more sophisticated X-ray imaging techniques allow for the recording of time-resolved data of bubbly rising dynamics (Verma et al., 2014). However, all of these techniques exhibit specific limitations. Intrusive probes interfere with the granular flow (Werther, 1999), pseudo-2D systems are dominated by wall effects (Gabor, 1967), $\mathrm{X}$-ray tomography can only provide information about the spatial distribution of particles within the bed but cannot provide information about their velocity and PEPT can only track individual particles.

An alternative tomographic technique is magnetic resonance imaging (MRI), which has used previously to study 3D granular systems and multiphase reactors (Fukushima, 1999; Gladden, 2013; Gladden et al., 2007; Mueth et al., 2000). Until recently, however, a major shortcoming of MRI has been the limited temporal resolution of velocity measurements of granular systems. Recent advances in real-time MRI have enabled the concurrent measurement of the local density and velocity of the solid phase within 3D fluidized beds (Penn et al., 2017). The technique has been applied to study the bubbling behaviour and particle velocity in fluidized beds of dry particles (Penn et al., 2018) as well as in beds with small amounts of liquids (Boyce et al., 2018). In the present work, real-time MRI was used to probe the fluidization dynamics of a 3D fluidized bed that contains a horizontal tube internal insert. We studied the local fluidization near the insert for gas velocities below $U_{\mathrm{mf}}$, and quantified particle velocity fluctuations and the distribution of gas bubbles around the tube insert for $U>U_{\mathrm{mf}}$.

\section{Methods}

\section{Magnetic resonance imaging}

All MRI measurements reported here were acquired with a clinical 3T human MRI system (Philips Achieva, Philips Healthcare, The Netherlands) using a custom-built 16-channel radiofrequency receiver array (Penn et al., 2017). MR Data was reconstructed using algebraic image reconstruction (Pruessmann et al., 1999) and processed using MATLAB equipped with the MRecon library (Gyrotools LLC, Zurich, Switzerland). Central vertical slices through the cylindrical fluidized bed were acquired with a field of view of $200 \mathrm{~mm} \times 300 \mathrm{~mm}$ (horizontal $(x)$ and vertical $(y)$, respectively). Three types of MRI measurements were performed: a) particle density measurements 
to study gas bubble dynamics, b) particle velocity measurements and c) fluidization-sensitive MRI to detect locally fluidized regions within the bed. For a) and b), single-shot pulse sequences (Stehling et al., 1991) were used that exploit the Hermitian symmetry (Noll et al., 1991) in order to obtain a short data acquisition time $T_{\text {acq. }}$. Details of the MRI sequences used are described in (Penn et al., 2017).

Gas bubble measurements. For each experimental condition, 500 snapshots of particle density with a temporal resolution of $T_{\mathrm{acq}}=6 \mathrm{~ms}$ were acquired with a spatial resolution $(\Delta x \times \Delta y \times \Delta z)$ of $3 \mathrm{~mm} \times 3 \mathrm{~mm} \times 10 \mathrm{~mm}$ and a delay of $\Delta \mathrm{t}$ of $200 \mathrm{~ms}$ between consecutive frames. The value for $\Delta \mathrm{t}$ was slower compared to its fastest possible value of $7 \mathrm{~ms}$ in order to allow the magnetic magnetization to recover between consecutive excitation pulses, thereby increasing the signal to noise ratio $(S N R)$ of the MR images and thus the robustness of the subsequent image analysis. After acquisition, the MR images were thresholded using a value of $15 \%$ of the maximum intensity value of the image series to distinguish gas-phase pixels from particle-laden pixels. The choice of this threshold value is discussed in the Supplementary Material and in Figure S5. Subsequently, the area of the bubbles, $A_{b}$ and their respective center of mass were detected. A minimum bubble area $A_{\min }=2 \pi \Delta x \Delta y$ was used to exclude signal fluctuations from being interpreted as bubbles. The equivalent bubble diameter (corresponding to a circle of the same area), $d_{b u b}=\sqrt{\frac{4 A_{b}}{\pi}}$, was calculated and used in the analysis shown in Fig. 3. Partly erupted bubbles at the free surface of the bed were excluded from the analysis. The positions and size of the gas bubbles were used to create Figs. 3, 4 and 5 and figs. S3 and S4 and the Supplementary Video.

Particle velocity measurements. For each experimental condition, 1000 snapshots of instantaneous particle velocity were acquired with a temporal and spatial resolution $(\Delta x \times \Delta y \times \Delta z)$ of $21 \mathrm{~ms}$ and $3 \mathrm{~mm} \times 5 \mathrm{~mm} \times 15 \mathrm{~mm}$, respectively, a field of view of $200 \mathrm{~mm} \times 300 \mathrm{~mm}$. Phase contrast velocimetry was used to measure particle velocities up to $1.5 \mathrm{~m} \mathrm{~s}^{-1}$ in both the $x$ and $y$ direction. In order to exclude the gas phase from the velocity analysis, the velocity data were thresholded using the same threshold value of $15 \%$ used for the particle position measurements. The temporal mean and the standard deviation of the velocity time series were calculated and are plotted in Figure 2.

Fluidization-sensitive MRI. These measurements were used to produce the data for Fig. 1. For each experimental condition, one time-averaged MR image was acquired over the course of $9 \mathrm{~s}$ 
with a spatial resolution $(\Delta x \times \Delta y \times \Delta z)$ of $2 \mathrm{~mm} \times 2 \mathrm{~mm} \times 10 \mathrm{~mm}$. Fluidized areas exhibit lower signal intensity (darker contrast) compared to unfluidized (stationary) areas when imaged with a fluidization-sensitive MRI pulse sequence. Details of this fluidization-sensitive MRI contrast can be found in (Penn et al., 2017). The basic principle of the contrast mechanism can be explained as follows: Fluidized particles exhibit an incoherent motion. This incoherent particle motion causes an attenuation of the MRI signal due to the dephasing of excited spins when exposed to a bipolar magnetic field gradient (in this case the prephaser and the first half of the readout gradient). Similar concepts are used in clinical MRI application to measure diffusion and perfusion in biological tissue (Le Bihan et al., 1986).

\section{Fluidized bed}

An acrylic cylinder of diameter $190 \mathrm{~mm}$ and height $300 \mathrm{~mm}$ was used as the fluidized bed. An acrylic plate of thickness $10 \mathrm{~mm}$ with 6416 laser cut holes of diameter $0.5 \mathrm{~mm}$ was used as the distributor. Gas was injected into a windbox of diameter $190 \mathrm{~mm}$ and height $150 \mathrm{~mm}$. The gas flow was controlled with a mass flow controller (F-203AV, Bronkhorst High-Tech B.V.) connected to a computer running LabView, (National Instruments Corporation). The pressure drop across the distributor was more than ten times the pressure drop across the bed for all of the gas flow rates studied here, thereby ensuring a homogeneous injection of air. Further details of the fluidized bed setup can be found in (Penn et al., 2018, 2017). Horizontal acrylic tubes of length $190 \mathrm{~mm}$ and diameter $30 \mathrm{~mm}$ and $40 \mathrm{~mm}$ (referred to herein as small and large tube, respectively), and wall thickness $3 \mathrm{~mm}$ were mounted into the fluidized bed at a height of $80 \mathrm{~mm}$ above the distributor. The bed was filled up to an unfluidized height of $160 \mathrm{~mm}$ with engineered spherical agar particles with a MRI-detectable medium chain triglyceride oil. The particles had a diameter of $1.02 \pm 0.12$ $\mathrm{mm}$, density of $1040 \mathrm{~kg} \mathrm{~m}^{-3}$, an angle of repose of $28 \pm 2^{\circ}$, a friction coefficient of 0.54 and a coefficient of restitution of $0.7 \pm 0.03$ (Penn et al., 2017). The inserts were also filled with particles in order to minimize the magnetic susceptibility mismatch between the air and the particle phase and thus assure acceptable image quality in regions close to the inserts. The procedure for measuring the minimum fluidization velocity $U_{\mathrm{mf}}$ is described in the Supplemental Information and depicted in Fig S1. 


\section{Results and Discussion}

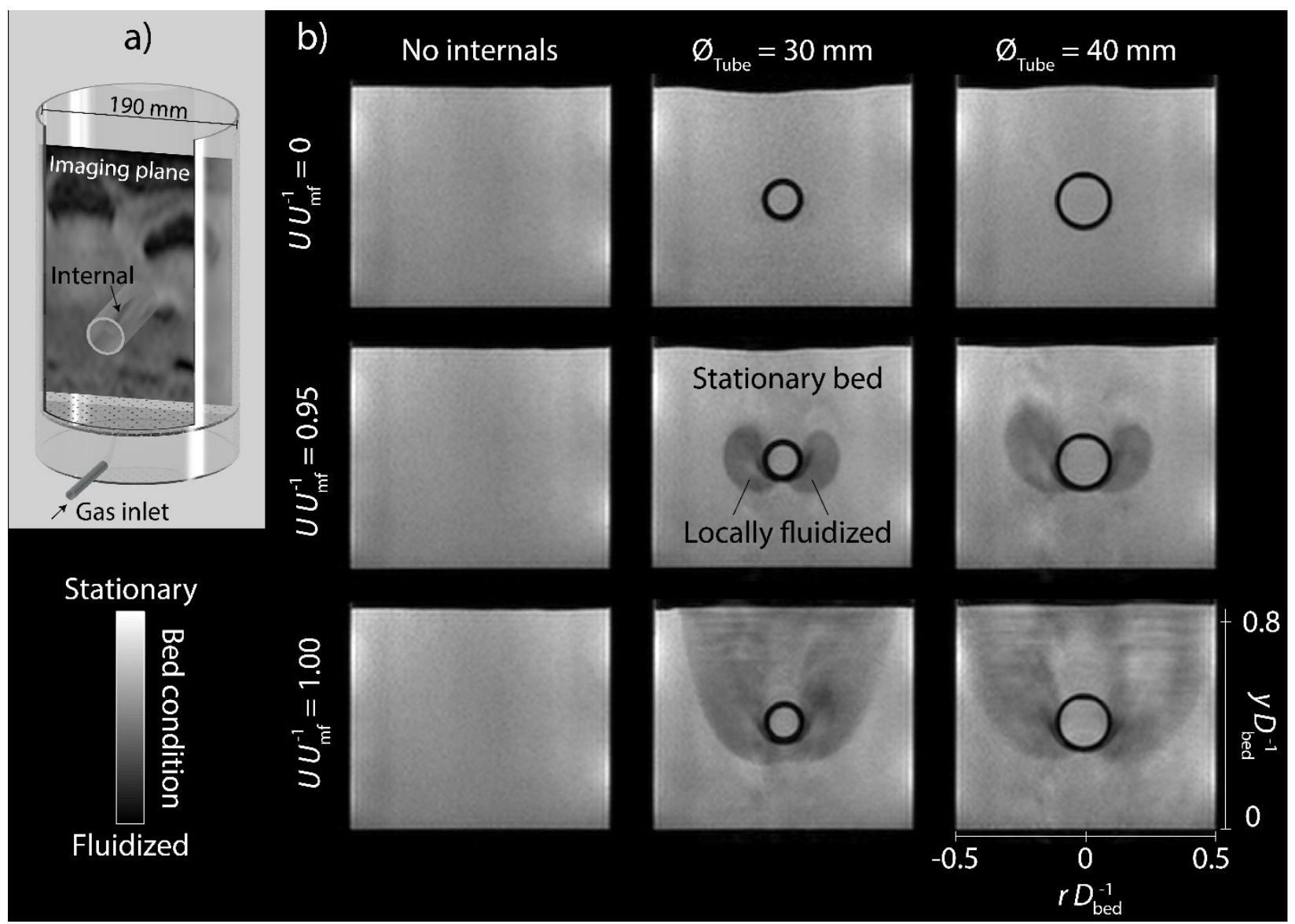

Figure 1. (a) Sketch of the cylindrical fluidized bed with a horizontal tube internal. (b) MR images of the particle bed for different gas flow rates (rows) and for beds without internals (left column) and with the small and large tube internal (center and right column, respectively). Locally fluidized and bubbling regions can be observed adjacent to the internals.

\section{Local fluidization and bubbling around an insert for $U<U_{\text {mf }}$}

Figure $1 \mathrm{~b}$ shows fluidization-sensitive MR images of beds with and without an inserted tube for gas velocities $U$ that are smaller than the minimum fluidization velocity $U_{\mathrm{mf}}$. Areas of local fluidization and bubbling (dark contrast) can be observed at the sides of the insert. The size of these areas increases with the diameter of the inserted tube and the gas velocity $U$. At $U=0.95 U_{\mathrm{mf}}$ (second row of Fig. 1b) the local fluidization and bubbling is confined to two kidney-shaped regions surrounding the insert, while for a higher gas velocity $U=1.00 U_{\mathrm{mf}}$ (lower row in Fig $1 \mathrm{~b}$ ), these regions expand to form a crater-shaped region that spans the area between the insert and the free surface. This local fluidization in the vicinity of the insert suggest that $U_{\mathrm{mf}}$ is exceeded in 
regions close to the obstacle where gas has to flow around it causing the local formation of bubbles around the lower part of the obstacle. This finding agrees with previous works reporting a preferential formation of gas bubbles in the vicinity of internals (Glass and Harrison, 1964) Interestingly, an unfluidized cap region can be observed above the tube, this region is approximately as wide as the obstacle diameter. This effect might be relevant for industrial processes that operate at superficial velocities close to $U_{\mathrm{mf}}$, such as the separation of solids based on density and size differences (Wu and Baeyens, 1998; Yoshida et al., 2010).

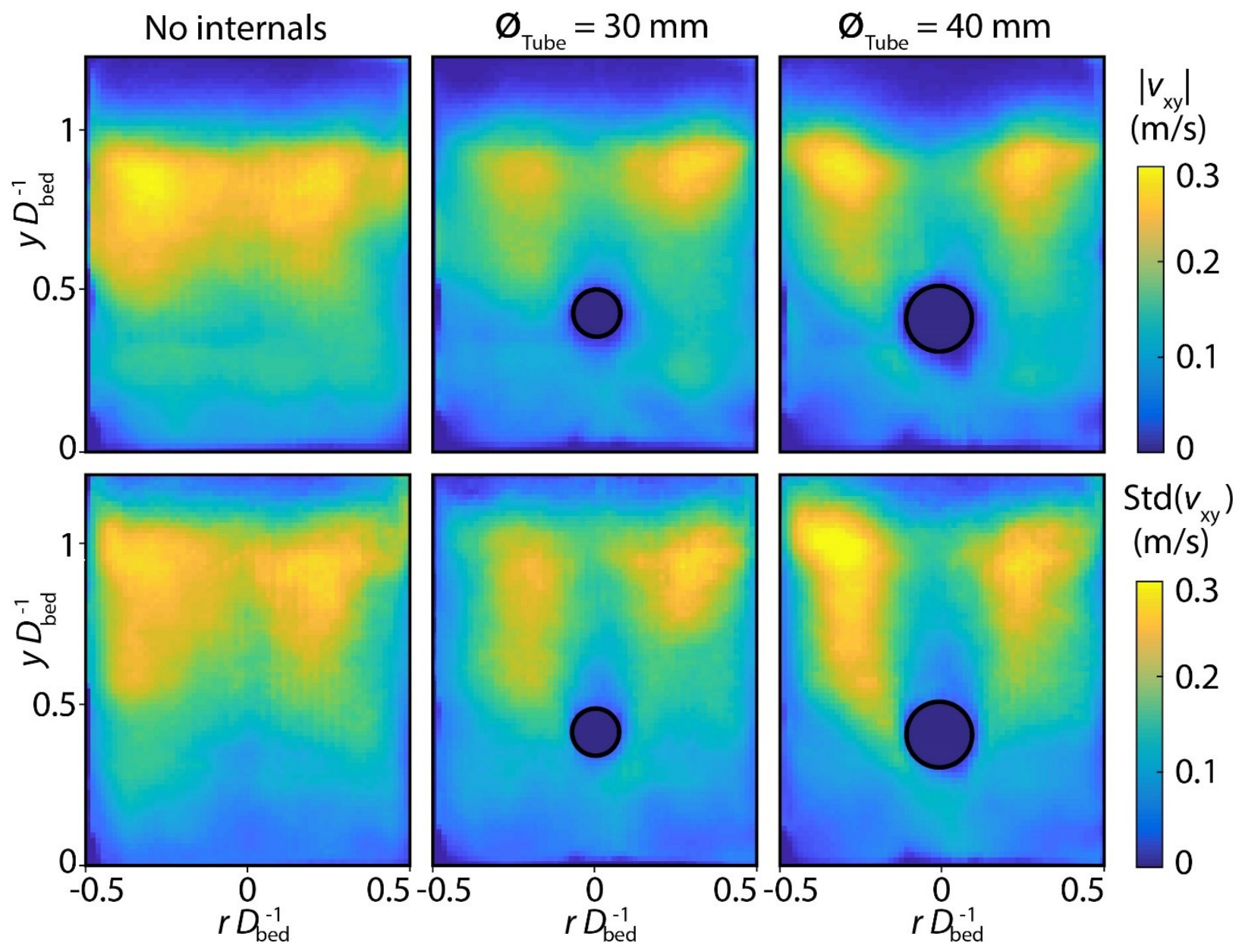

Figure 2. Time-averaged in-plane velocity (upper row) and velocity fluctuations (lower row) of the particle-laden phase based on a series of 1000 velocity encoded phase-contrast MRI images acquired within a total time of $24 \mathrm{~s}$ for each experimental condition. Data is shown for a gas velocity of $U / U_{\mathrm{mf}}=1.8$. Figure S2 shows the same analysis for a gas flow rate of $U / U_{\mathrm{mf}}=1.4$. Reduced particle velocity and velocity fluctuations can be observed in the wake region above the internals, indicating a reduced mobility of the particle phase in these regions. A slight asymmetry about the central vertical axis can be observed, likely caused by the short sampling time or tiny inhomogeneities of the distributor. The light half circle patterns that are visible in the velocity data at the bottom of the bed as well as the light horizontal line of apparently reduced particle velocity, 
adjacent to the left side of the obstacles are artifacts of the parallel MRI reconstruction and thus should be ignored.

\section{Fluidization dynamics for $U>U_{\mathrm{mf}}$}

Figure 2 shows the temporally averaged particle velocity (upper row) and the corresponding particle velocity fluctuations (lower row) for a gas flow rate $U / U_{\mathrm{mf}}=1.8$. The left column of Fig. 2 shows the fluidized bed without internals, while the center and right columns show beds with the small and the large tube inserts, respectively. Above the tube insert, both the particle velocity and their fluctuations are lower compared to the reference bed without internals indicating a reduced particle mobility. The horizontal extent of these regions of reduced particle mobility is approximately equal to the tube diameter, while its vertical extent spans from the top of the obstacle up to the free surface of the bed. This region above the insert is herein referred to as the wake region. The same analysis for $U / U_{\mathrm{mf}}=1.4$ is displayed in the Supplementary Figure $\mathrm{S} 2$. The above observations hold for both gas velocities studied. However, for $U / U_{\mathrm{mf}}=1.8$, the width of the wake region is slightly reduced with decreasing distance to the free surface of the bed indicating that the region of reduced particle mobility in the wake of internals becomes smaller for higher gas velocities. A de-fluidized cap in the wake of internals has been previously observed in 2D systems and postulated in 3D systems (Hager and Schrag, 1976; Rao and Muller, 2013). These works identified the absence of bubbles in these regions to cause the de-fluidized cap phenomenon, since bubbles drive solid motion (Müller et al., 2007). In order to test these previous observations, here we also detected gas bubbles and compared their size and spatial distribution in the fluidized bed to the particle velocity data. 


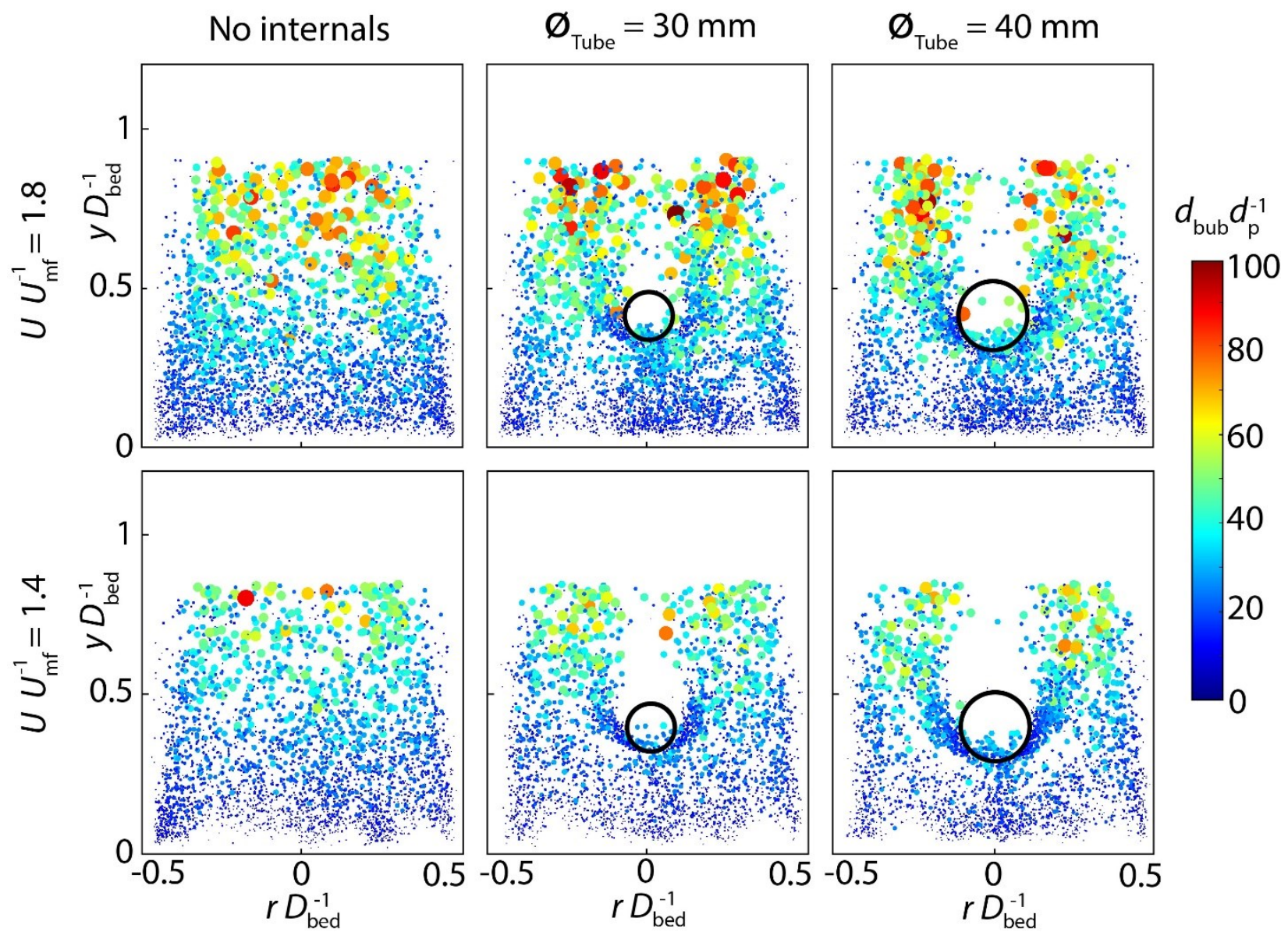

Figure 3. Size (color and diameter of the dots) and spatial distribution of the center of mass of gas bubbles in the fluidized bed. The upper and lower rows show data for gas velocities of $U / U_{\mathrm{mf}}=1.8$ and 1.4, respectively. Region of strongly reduce bubble occurrence can be observed in the wake region above the internals, indicating reduced gas-solid mixing in these regions. These regions coincide agree well with the regions of reduce particle mobility shown in Fig. 2.

Figure 3 shows the position and size of gas bubbles in beds for gas velocities $U / U_{\mathrm{mf}}=1.4$ (lower row) and 1.8 (upper row). As in Figure 2, the left column displays the bed without internals, the center and right columns show the beds with the small and large tube insert, respectively. The supplementary video displays the MR image series used for this analysis. Each dot in the figure represents the position of the center of mass of a gas bubble, while its color and size depends on the equivalent diameter of the bubble. In agreement with previous studies (Darton et al., 1977; Penn et al., 2018; Werther, 1977), the bubble size increases monotonically with the vertical position $(y)$ in a fluidized bed without internal inserts, while the number of bubbles decreases. When a tube is inserted into the bed, the bubble motion is affected in the following ways: a) Bubbles are diverted 
to both sides of the insert leading to a strongly reduced number of bubbles in the wake region of the insert and $\mathrm{b}$ ) the center region (around $r=0$ ) below the tube shows a higher number of bubbles. These observations are valid for both gas velocities studied here, i.e. $U / U_{\mathrm{mf}}=1.4$ and 1.8. It is important to note that some of the colored dots in Figure 3 are located within the obstacles which might give the impression that some bubbles penetrate these obstacles. However, this is not the case: if a bubbles wraps around the obstacles, the bubble's center of mass can be located within the impenetrable obstacle even if no part of the bubble is located within the obstacle.

The qualitative observations discussed above were further quantified in Figures 4 and 5 for a gas velocity of $U / U_{\mathrm{mf}}=1.8$. The very same analyses for $U / U_{\mathrm{mf}}=1.4$ can be found in the supplementary Figures S3 and S4. Figure 4 and 5 show, respectively, the horizontal and vertical profiles of the number of bubbles per frame and unit area, $N_{\mathrm{b}}$ (panels a and $\mathrm{b}$ ) and their mean bubble diameter (panels c and d). Fig. 4e and Fig. 5e display the spatial position of these profiles for clarity.
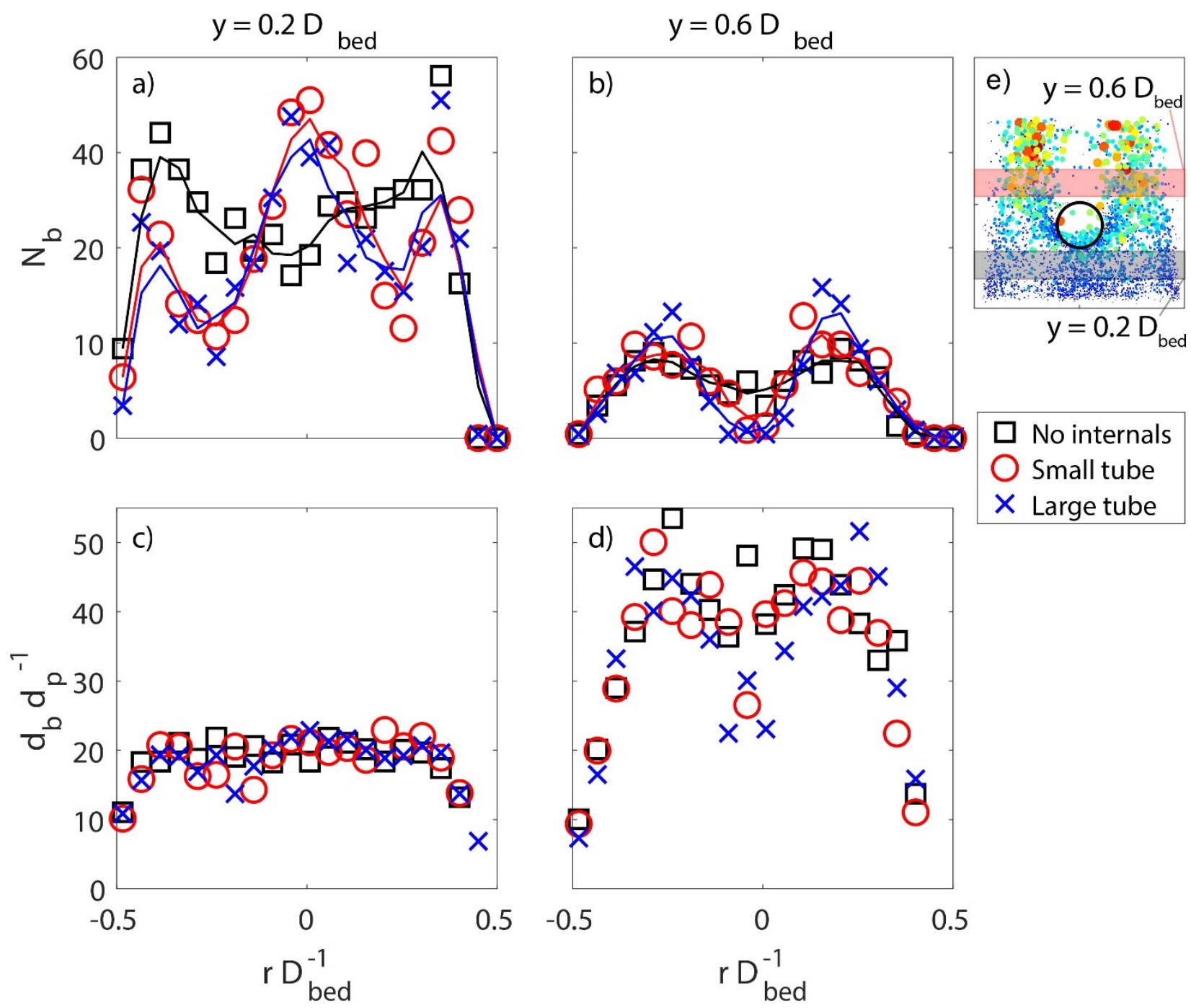
Figure 4. Horizontal profiles of mean number of bubble per image and unit area $(a, b)$ and mean equivalent circular diameter of the gas bubbles. The solid lines represent a moving average with a filter width of three data points. (c, d) for $U / U_{m f}=1.8$. Panels (a) and (c) cover a region below the internals at $y=0.2 D_{\text {bed, }}$ while panels (b) and (d) cover a region above the internals at $y=0.6 D_{\text {bed, }}$ as displayed in (e).

Figure 4 plots horizontal profiles of the number of bubbles and their mean diameter at two different vertical positions, viz. below the obstacle at $y=0.2 D_{\text {bed }}($ Fig. 4 a,c) and above the obstacle at $y=$ $0.6 D_{\text {bed }}$ (Fig. 4 b,d). Below the insert, the number of bubbles follows a "W-shaped" profile with an increased number of bubbles in the center region of the bed and two lateral dips at approximately $r=0.25 D_{\text {bed }}$ (Fig. 4a). These findings suggest that there is an increased rate of bubble formation in the region below the insert. To the best knowledge of the authors, this trend has not been observed previously. The physical reason for the increased rate of bubble formation below the insert could not be found here and is worthy of further investigation. Above the insert, the shape of the profile is inverted to an "M-shape" that exhibits a strong dip in its center (corresponding to the wake region at $r=0$ ) and two peaks at around $r=0.25 D_{\text {bed }}$ (Fig. $4 \mathrm{~b}$ ). This occurs due to bubbles being diverted by the insert. Interestingly, the mean bubble size, does not change with the presence of internals, except for a the wake region, where only a small number of bubbles were found in beds with internals (Fig. 4 c,d). 

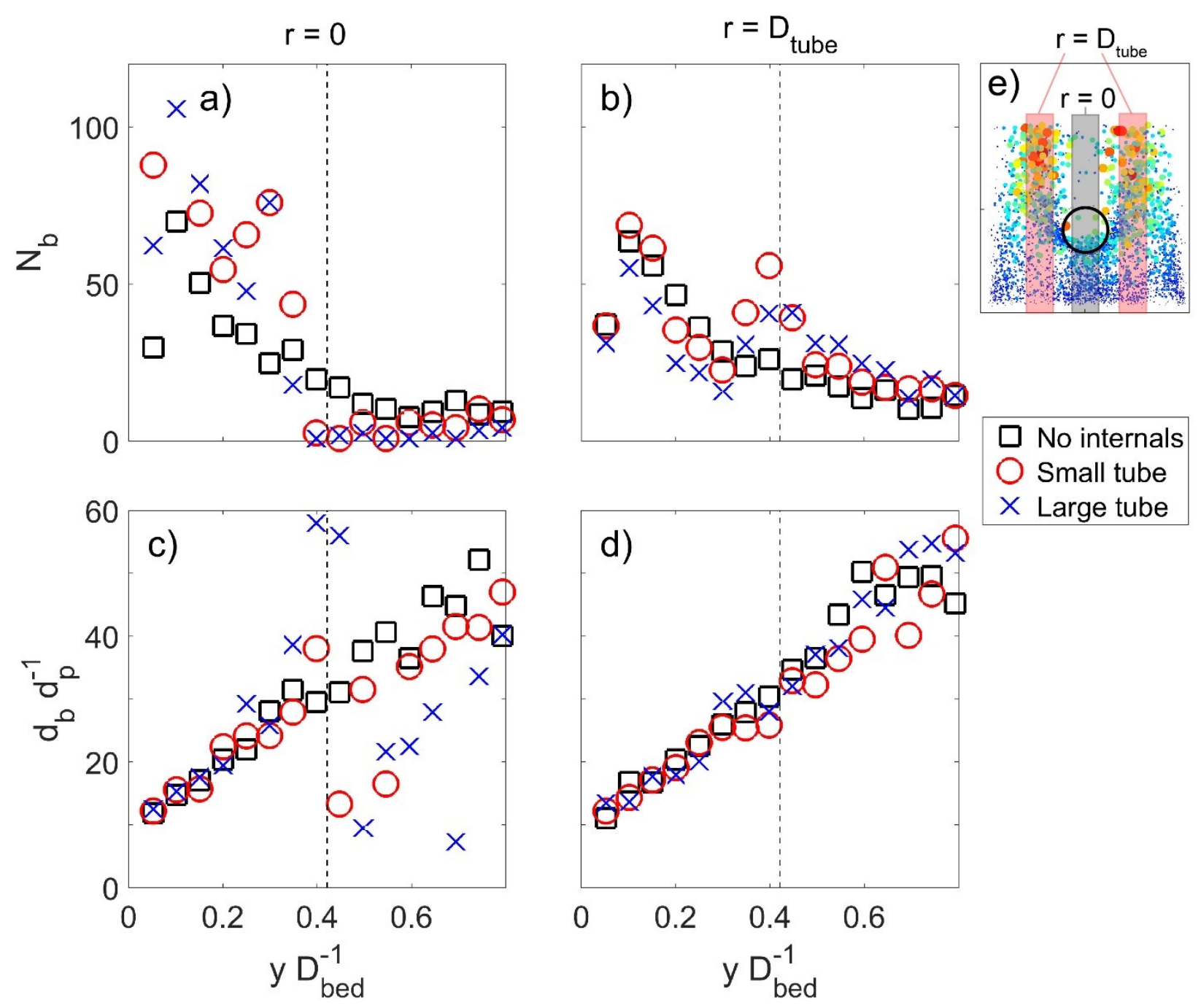

Figure 5. Vertical profiles of mean number of bubble per image and unit area (a, b) and mean equivalent circular diameter of the gas bubbles (c, d) for $U / U_{m f}=1.8$. Panels (a) and (c) cover the center region at $r=0$, while panels (b) and (d) cover a the lateral region at $r=D_{\text {tube, }}$ as displayed in $(\mathbf{e})$.

Figure 5 shows vertical profiles of the number of bubbles and their mean diameter at two different radial positions: along the center of the bed at $r=0$ (Fig. 5 a, c) and at $r=D_{\text {tube }}$ (Fig. 5 b, d). The dashed vertical line represents the position of the center of the tubes. The most prominent observation is the strongly reduced number of bubbles in the wake region of the obstacles (right hand side of the dashed line in Fig. 5a) compared to the reference bed without internals. At the same time, the few bubbles that penetrate the wake region above the insert are significantly smaller as compared to the reference bed. Below the insert (at the left hand side of the dashed line in Fig. $5 \mathrm{a}$ ), the number of bubbles is increased, while the mean bubble size (Fig. 5c, left) does not exhibit 
a substantial deviation from the reference bed, except for regions directly below the insert. These observations indicate that inserts do affect fluidization dynamics not only in their wake region, but also in regions upstream of the insert. We observe a higher density of bubbles in the upper lateral regions of a bed with inserts (right side of Fig. 5b), most likely due to the deviation of central gas bubbles by the insert. As observed previously, the mean bubble diameter in these lateral regions is not affected by the inserts (Fig. 5d)

\section{Conclusion}

Instantaneous MR images of particle velocity and particle position were acquired to study the effect of an internal tube insert on fluidization and bubble dynamics in 3D fluidized beds. For gas velocities $U<U_{\mathrm{mf}}$, local fluidization and bubbling were observed in areas around the insert, likely caused by the gas velocity exceeding $U_{\mathrm{mf}}$ locally. These locally fluidized areas increased in size with increasing insert diameter. For gas velocities $U>U_{\mathrm{mf}}$, internals were found to increase the heterogeneity of the fluidization dynamics within the bed. The wake region above the insert exhibited a reduced particle mobility and a reduced number and size of gas bubbles, while the region directly below the insert and the upper lateral regions outside the wake of the insert, exhibited an increased number of bubbles. The size of the region of reduced bed mobility was approximately as wide as the internal tube, and it reached from the top of the insert to the free surface of the bed.

The findings presented in this work are anticipated to be helpful for improving the design of fluidized bed reactors, for applications in which the homogeneity of fluidization is essential for product or operational quality. In particular, for cases in which internals are used to control the temperature in the reactor, our findings suggest that due to the reduced particle mobility, the heat transfer rate from the insert to the particle phase might be reduced in the wake region of the insert with the possibility of the formation of hot spots. To summarize, the MRI methodology used in this work provided valuable insights into the dynamics of 3D fluidized beds with internals. Future work will assess the effect of internals and baffles of different shapes, sizes and orientations as well as the effect of multiple internals across a wider range of gas velocities. 


\section{Acknowledgements}

This work was supported by the Swiss National Science Foundation under grant no. 200021_153290. The authors declare that they do not have any competing interests.

\section{References}

Baskakov, A.P., Berg, B.V., Vitt, O.K., Filippovsky, N.F., Kirakosyan, V.A., Goldobin, J.M., Maskaev, V.K., 1973. Heat transfer to objects immersed in fluidized beds. Powder Technology 8, 273-282. https://doi.org/10.1016/0032-5910(73)80092-0

Boyce, C.M., Penn, A., Pruessmann, K.P., Müller, C.R., 2018. Magnetic resonance imaging of gas-solid fluidization with liquid bridging, in: AIChE Journal. pp. 2958-2971. https://doi.org/10.1002/aic.16036

Darton, R.C., LaNeuze, R.D., Davidson, J.F., Harrison, D., 1977. Bubble growth due to coalesence in fluidised beds. Trans. Inst. Chem. Eng. 55, 274-280.

Franka, N.P., Heindel, T.J., 2009. Local time-averaged gas holdup in a fluidized bed with side air injection using X-ray computed tomography. Powder Technology 193, 69-78. https://doi.org/10.1016/J.POWTEC.2009.02.008

Fukushima, E., 1999. Nuclear magnetic resonance imaging as a tool to study flow. Annual Review of Fluid Mechanics 31, 95-123.

Gabor, J.D., 1967. Wall effects on fluidized particle movement in a two-dimensional column, in: International Symposium on Fluidization, Eindhoven. pp. 230-241.

Gladden, L.F., 2013. Magnetic resonance in reaction engineering: Beyond spectroscopy. Current Opinion in Chemical Engineering 2, 331-337. https://doi.org/10.1016/j.coche.2013.05.005

Gladden, L.F., Anadon, L.D., Dunckley, C.P., Mantle, M.D., Sederman, A.J., 2007. Insights into gas-liquid-solid reactors obtained by magnetic resonance imaging. Chemical Engineering Science 62, 6969-6977. https://doi.org/10.1016/j.ces.2007.08.084

Glass, D., Harrison, D., 1964. Flow patterns near a solid obstacle in a fluidized bed. Chemical Engineering Science 19, 1001-1002.

Hager, W.R., Schrag, S.D., 1976. Particle circulation downstream from a tube immersed in a fluidized bed. Chemical Engineering Science 31, 657-659. https://doi.org/10.1002/jor.22593

Kunii, D., Levenspiel, O., 1991. Fluidization Engineering, 2nd ed. Butterworth-Heinemann.

Laverman, J.A., Roghair, I., Annaland, M. van S., Kuipers, H., 2008. Investigation into the hydrodynamics of gas-solid fluidized beds using particle image velocimetry coupled with digital image analysis. The Canadian Journal of Chemical Engineering 86, 523-535. https://doi.org/10.1002/cjce.20054

Le Bihan, D., Breton, E., Lallemand, D., Grenier, P., Cabanis, E., Laval_Jeantet, M., 1986. MR imaging of Intravoxel Incoherent Motions: Application to Diffusion and Perfusion in Neurologic Disorders. Radiology 161, 401-407.

Mueth, D.M.D., Debregeas, G.G.F., Karczmar, G.G.S., Eng, P., Nagel, S.R., Jaeger, H., 2000. Signatures of granular microstructure in dense shear flows. Nature 3, 385-389. https://doi.org/10.1038/35019032

Müller, C.R., Davidson, J.F., Dennis, J.S., Hayhurst, A.N., 2007. A study of the motion and eruption of a bubble at the surface of a two-dimensional fluidized bed using Particle image velocimetry (PIV). Industrial and Engineering Chemistry Research 46, 1642-1652. https://doi.org/10.1021/ie0611397 
Noack, R., 1970. Lokaler Wärmeübergang an horizontalen Röhren in Wirbelschichten. Chemie Ingenieur Technik 42, 371-376. https://doi.org/10.1002/cite.330420610

Noll, D.C., Nishimura, D.G., Macovski, A., 1991. Homodyne Detection in Magnetic Resonance Imaging. IEEE Transactions on Medical Imaging 10, 154-163. https://doi.org/10.1109/42.79473

Penn, A., Boyce, C.M., Kovar, T., Tsuji, T., Pruessmann, K.P., Müller, C.R., 2018. Real-Time Magnetic Resonance Imaging of Bubble Behavior and Particle Velocity in Fluidized Beds. Industrial \& Engineering Chemistry Research 57, 9674-9682. https://doi.org/10.1021/acs.iecr.8b00932

Penn, A., Tsuji, T., Brunner, D.O., Boyce, C.M., Pruessmann, K.P., Müller, C.R., 2017. Real-time probing of granular dynamics with magnetic resonance. Science Advances 3, e1701879. https://doi.org/10.1126/sciadv.1701879

Pruessmann, K.P., Weiger, M., Scheidegger, M.B., Boesiger, P., 1999. SENSE: Sensitivity encoding for fast MRI. Magnetic Resonance in Medicine 42, 952-962. https://doi.org/10.1002/(SICI)1522-2594(199911)42:5<952::AID-MRM16>3.0.CO;2-S

Rao, P., Muller, M., 2013. Characterization of de-fluidized regions in the wake of submerged objects in a small particle fluidized bed. Powder Technology 243, 53-58. https://doi.org/10.1016/J.POWTEC.2013.03.044

Senadeera, W., Bhandari, B.R., Young, G., Wijesinghe, B., 2003. Influence of shapes of selected vegetable materials on drying kinetics during fluidized bed drying. Journal of Food Engineering 58, 277-283. https://doi.org/10.1016/S0260-8774(02)00386-2

Stehling, M.K., Turner, R., Mansfield, P., 1991. Echo-Planar Imaging: Magnetic Resonance Imaging in a Fraction of a Second. Science (New York, N.Y.) 254, 43-50.

Van de Velden, M., Baeyens, J., Seville, J.P.K., Fan, X., 2008. The solids flow in the riser of a Circulating Fluidised Bed (CFB) viewed by Positron Emission Particle Tracking (PEPT). Powder Technology 183, 290-296. https://doi.org/10.1016/J.POWTEC.2007.07.027

Verma, V., Padding, J.T., Deen, N.G., Kuipers, J.A.M.H., Barthel, F., Bieberle, M., Wagner, M., Hampel, U., 2014. Bubble dynamics in a 3-D gas-solid fluidized bed using ultrafast electron beam X-ray tomography and two-fluid model. AIChE Journal 60, 1632-1644. https://doi.org/10.1002/aic. 14393

Werther, J., 1999. Measurement Techniques in Fluidized Beds. Powder Technology 102, 15-36. https://doi.org/10.1016/S0032-5910(98)00202-2

Werther, J., 1977. Zur Problematik der Massstabsvergrösserung von Wirbelschichtreaktoren. Chem.-lng.-Tech. 49, 777-785.

Werther, J., Molerus, O., 1973. The local structure of gas fluidized beds - I. A statistically based measuring system. International Journal of Multiphase Flow 1, 103-122. https://doi.org/10.1016/0301-9322(73)90007-4

Wu, S.Y., Baeyens, J., 1998. Segregation by size difference in gas fluidized beds. Powder Technology 98, 139-150. https://doi.org/10.1016/S0032-5910(98)00026-6

Yasuo, K., Hiroshi, I., Kiyoshi, T., 1988. Fluidization and heat-transfer characteristics around a horizontal heated circular cylinder immersed in a gas fluidized bed. International Journal of Heat and Mass Transfer 31, 349-358. https://doi.org/10.1016/0017-9310(88)90017-8

Yoshida, M., Nakatsukasa, S., Nanba, M., Gotoh, K., Zushi, T., Kubo, Y., Oshitani, J., 2010. Decrease of $\mathrm{Cl}$ contents in waste plastics using a gas-solid fluidized bed separator. Advanced Powder Technology 21, 69-74. https://doi.org/10.1016/j.apt.2009.11.002 


\section{Supplementary Information}

The Supplementary Information contains additional details about the determination of the minimum fluidization velocity and the choice of the threshold value. It further contains the following:

- Supplementary Video available online at https://doi.org/10.1016/j.ces.2018.12.041

- Figure S1. Determination of the minimum fluidization velocity Umf.

- Figure S2. Time-averaged in-plane velocity and velocity fluctuations for $U / U_{\mathrm{mf}}=1.4$.

- $\quad$ Figure S3. Horizontal profiles of mean number and size of gas bubbles for $U / U_{\mathrm{mf}}=1.4$.

- $\quad$ Figure S4. Vertical profiles of mean number and size of gas bubbles for $U / U_{\mathrm{mf}}=1.4$.

- Figure S5. The effect of the threshold value on the results of this study.

\section{Minimum fluidization velocity $\boldsymbol{U}_{\mathrm{mf}}$}

The minimum fluidization velocity $U_{\mathrm{mf}}$ of the particles in the fluidized bed reactor was determined using a Honeywell 40PC001B1A pressure sensor that was connected via a rubber hose to a hollow brass tube of outer diameter $2 \mathrm{~mm}$, which was inserted into the fluidized bed. The bottom opening of the rod was closed using solder tin and a tiny hole of diameter $0.2 \mathrm{~mm}$ was drilled into the brass rod from the side at $10 \mathrm{~mm}$ distance from the soldered bottom in order to mitigate local inflow effects near the distributor. The sensor was powered by a 5V DC supply, smoothed with a large capacitor the decrease the power grid noise and fed into the analog input of a NI DAQ 6009 data acquisition card (National Instruments). The sensor was calibrated using a pressure calibration device, KAL 84 (Halstrup Walcher). Pressure difference between the ambient pressure and the bottom of the fluidized bed were plotted against the gas flow $U / U_{\mathrm{mf}}$ as shown in Figure $\mathrm{S} 1$. In each

of three consecutive experimental runs, the gas flow was decreased from $U / U_{\mathrm{mf}}=1.4$ to 0 at a rate of $\Delta U=-5 \times 10^{-3} U_{\mathrm{mf}} \mathrm{s}^{-1}$. The data were fitted for normalized pressure values between 0.50 and 0.85 times the gravitational pressure of the particle bed (red line in Fig. S1). Subsequently this fit was extrapolated and intersected with the line that corresponds the gravitational weight of the particle bed, $\Delta \mathrm{P} A_{\mathrm{bed}} w^{-1}=1$ (horizontal dashed line). The abscissa of the intersection point was used as $U_{\mathrm{mf}}$, for which a value of $0.25 \mathrm{~m} / \mathrm{s}$ was found. 


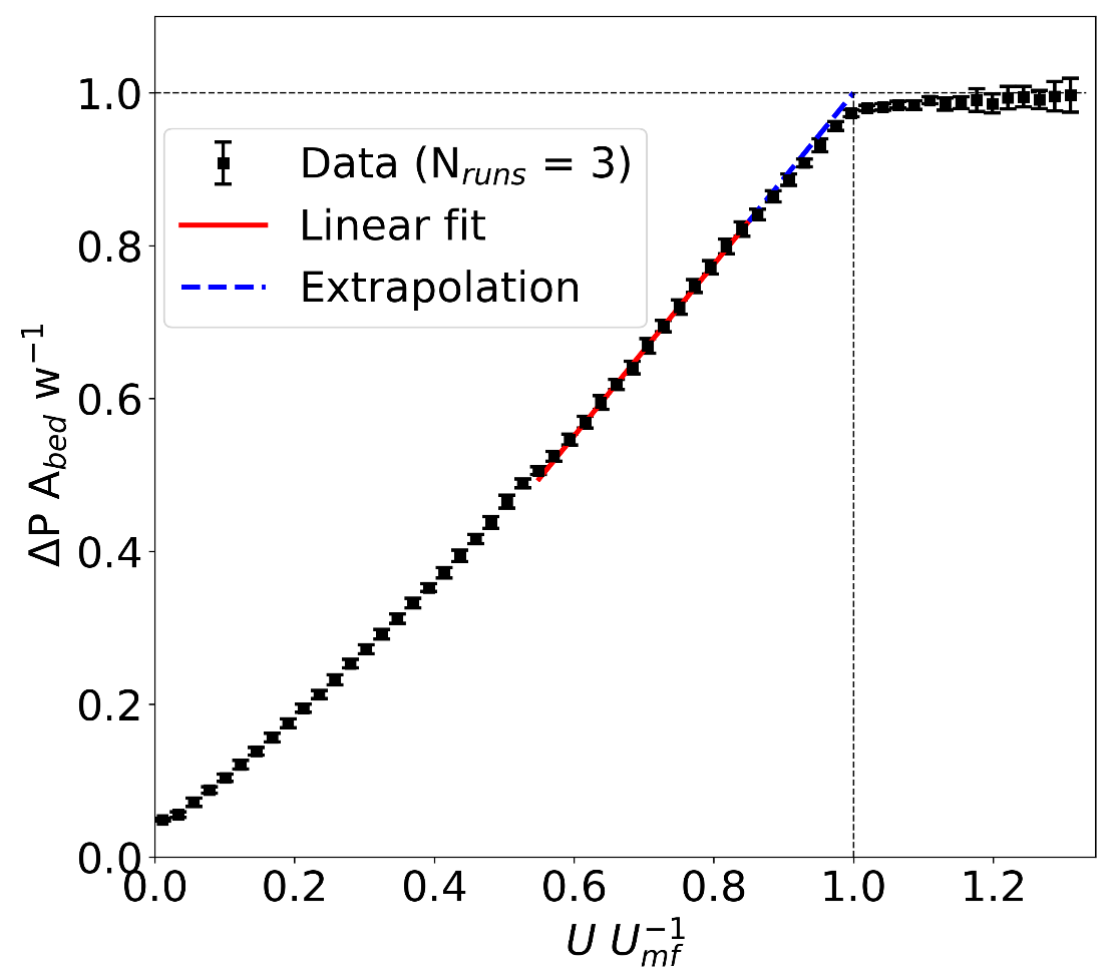

Figure S6. Determination of the minimum fluidization velocity $U_{\mathrm{mf}}$ by correlating the pressure drop $\Delta P$ between the bottom of the fluidized bed and the free surface with the gas flow rate $U$. 


\section{Time-averaged in-plane velocity and velocity fluctuations for $U / U_{m f}=1.4$}

The following Figure shows the temporally averaged particle velocity and velocity fluctuations for a gas flow rate $\mathrm{U} / \mathrm{Umf}=1.4$. The overall velocities are largely smaller compared to the higher gas flow rate of $\mathrm{U} / \mathrm{Umf}=1.8$, shown in Figure 2. However, the observed trends concerning the size of the region of reduced particle mobility in the wake of the obstacle hold for both flow rates studied here. At the same time, the asymmetry of data about the central vertical axis is increased for the lower flow rate, indicating an increased heterogeneity of the bed at lower gas flow rates.

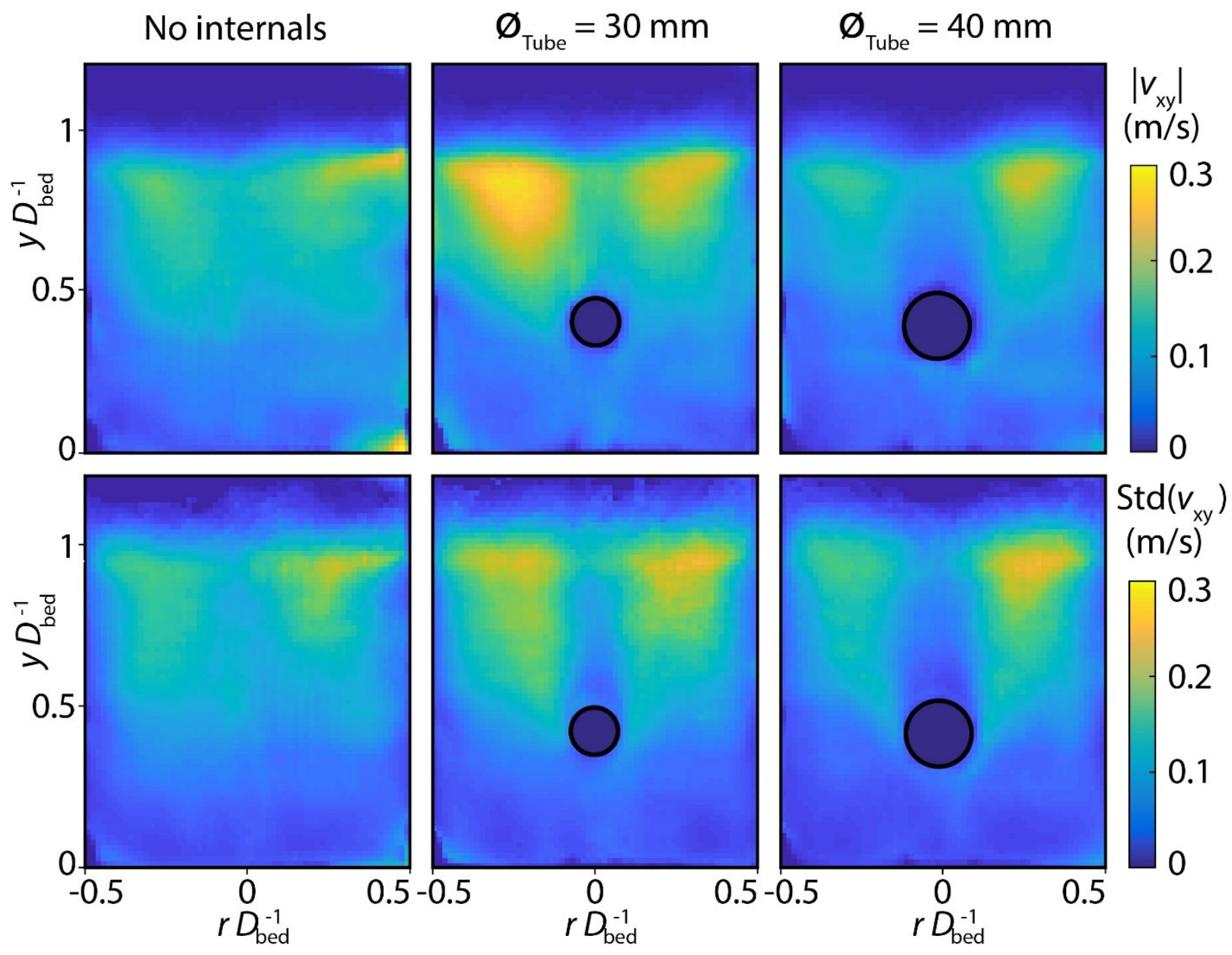

Figure S7. Time-averaged in-plane velocity (upper row) and velocity fluctuations (lower row) of the particle-laden phase based on a series of 1000 velocity encoded phase-contrast MRI images acquired within a total time of $24 \mathrm{~s}$ for each experimental condition. Data is shown for a gas velocity of $\mathrm{U} / \mathrm{Umf}=1.4$. Reduced particle velocity and velocity fluctuations can be observed in the wake region above the internals, indicating a reduced mobility of the particle phase in these regions. 


\section{Horizontal and vertical profiles of bubble number and bubble size for $U / U_{m f}=1.4$}

The following two Figures show the same analyses as shown in Figs. 4 and 5 but for a superficial gas velocity $U / U_{m f}=1.4$. The quantitative results differ compared to the case with higher gas velocity $U / U_{m f}=1.8$ (Figs. 4 and 5), however, the qualitative trends described in the main text hold for both cases studied in this work.
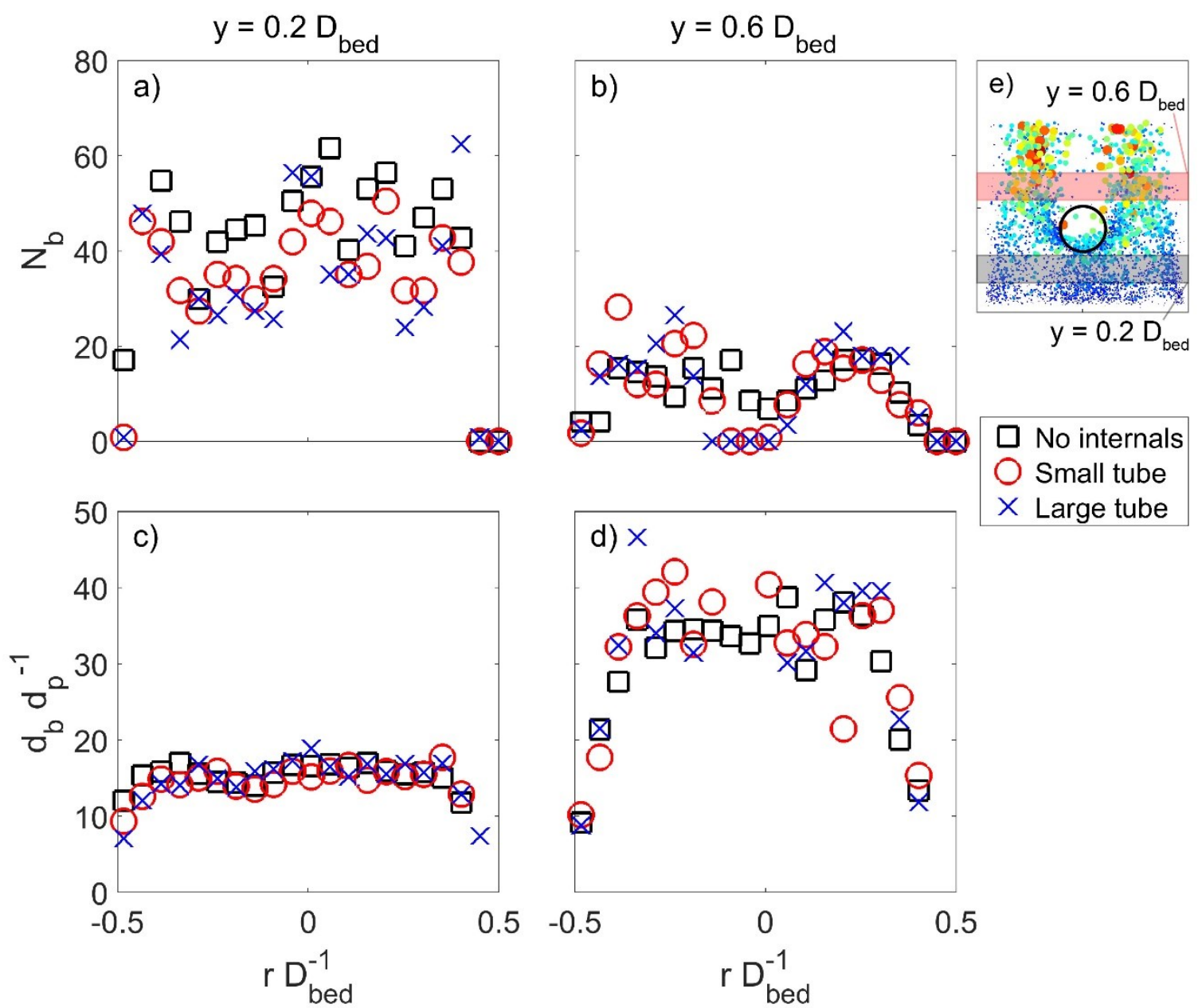

Figure S3. Horizontal profiles of mean number of bubbles per image and unit area $(a, b)$ and mean equivalent circular diameter of the gas bubbles (c, d) for $U / U_{m f}=1.4$. Panels (a) and (c) cover a region below the internals at $y=0.2 D_{\text {bed, }}$ while panels (b) and (d) cover a region above the internals at $y=0.6 D_{\text {bed, }}$ as displayed in (e). 

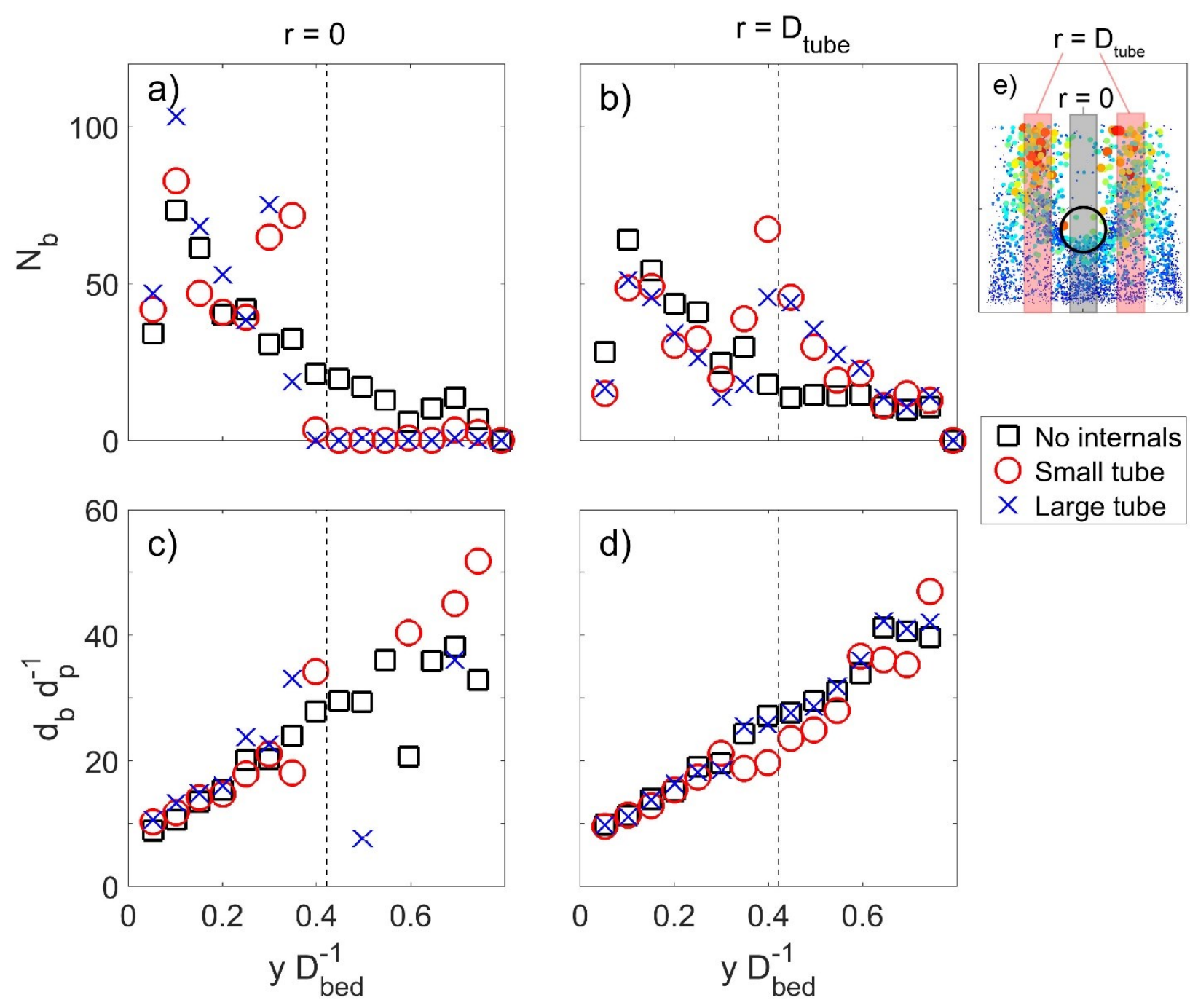

Figure S4. Vertical profiles of mean number of bubbles per image and unit area (a, b) and mean equivalent circular diameter of the gas bubbles (c, d) for $U / U_{m f}=1.4$. Panels (a) and (c) cover the center region at $r=0$, while panels (b) and (d) cover a the lateral region at $r=D_{\text {tube, }}$ as displayed in $(\mathbf{e})$.

\section{The effect of the threshold value on the results of this study}

For both particle density and particle velocity measurements, a threshold value of $15 \%$ of the maximum signal intensity of each image series was used to distinguish between gas phase and particle-laden phase. Figure S4 shows an MR particle density image prior to (Fig. S4a) and after thresholding with $10 \%, 15 \%$ and $20 \%$ (Fig. S4b). Based on an optical comparison between the thresholded image and the original image, the threshold value was determined. It can be observed that a threshold value of $10 \%$ is unable to detect smaller bubbles, while a value of $20 \%$ often detects neighboring bubbles as a single bubble. The sensitivity of the mean bubble size on the choice of this threshold value given shown in (Fig. S4c): Throughout all vertical positions an 
increase in threshold value decreases the detected mean bubble size monotonically, thus it does not affect the conclusions of this work.

a)

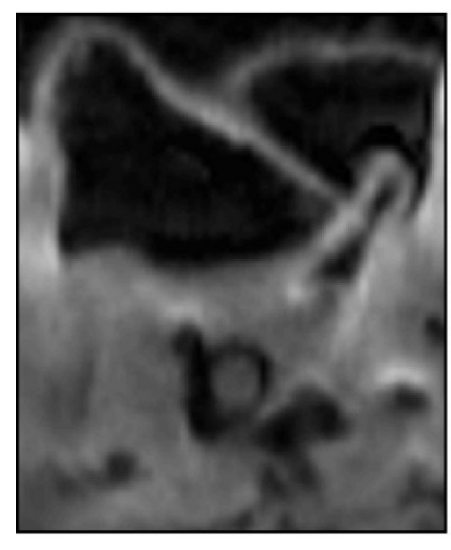

b)

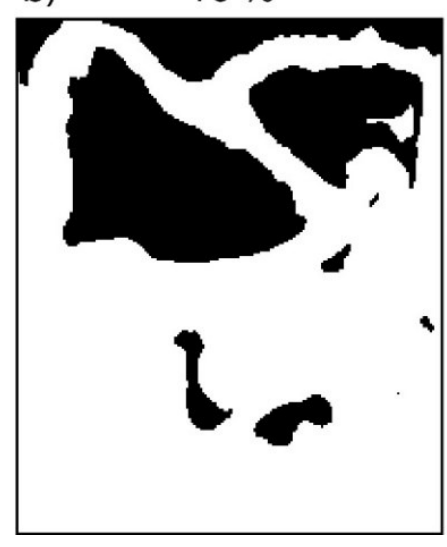

c)

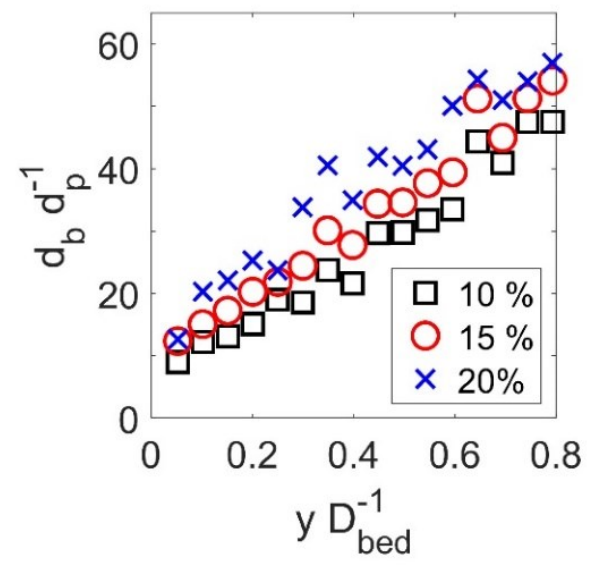

$15 \%$

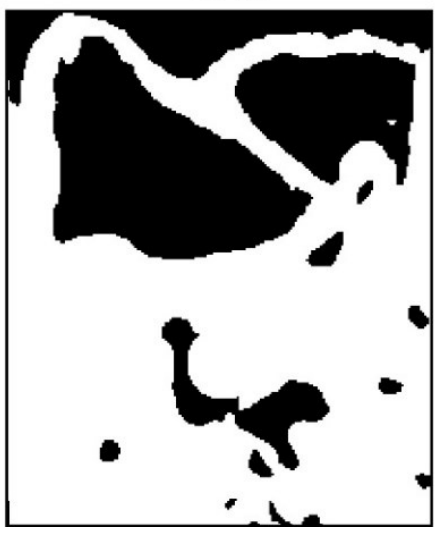

$20 \%$

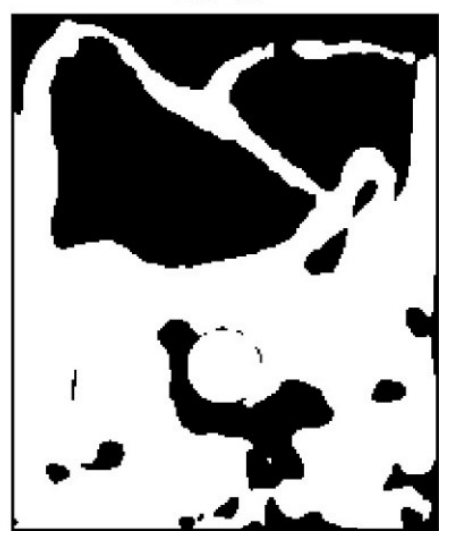

Figure S5. (a) Snapshot of an MR image series of the bubbling bed with small obstacle for a gas flow $U / U_{m f}=1.8$. (b) Binary image after applying a threshold of $10 \%, 15 \%$ and $20 \%$ of the maximum pixel intensity of the entire image series, respectively. (c) Effect of varying threshold value on mean bubble diameter across different vertical positions $y$ in the bed. 